PAPER

\title{
Separation Algorithm for Biosignals as Preprocess in Detecting Circulatory Disease
}

\author{
Kenji Hashiodani ${ }^{\# 1}$, Shinichi Takada ${ }^{\# 2}$, Yohei Fukumizu ${ }^{\# 3}$, Hironori Yamauchi ${ }^{\# 4}$, \\ Yoshimasa Kurumi ${ }^{* 5}$ and Tohru Tani ${ }^{* 6}$ \\ ${ }^{\#}$ Ritsumeikan University, 1-1-1 Noji Higashi, Kusatsu-shi, Shiga 525-8577, Japan \\ E-mail: $\left\{{ }^{1}\right.$ ri007060, ${ }^{2}$ ri0000kh $\} @$ ed.ritsumei.ac.jp \\ $\left\{{ }^{3}\right.$ fukumizu, ${ }^{4}$ yamauchi $\}$ se.ritsumei.ac.jp \\ *Shiga University of Medical Science, Seta Tsukinowa-cho, Otsu-shi, Shiga 520-2192, Japan \\ E-mail: $\left\{{ }^{5}\right.$ kurumi, $\left.{ }^{6} \tan \right\} @$ belle.shiga-med.ac.jp
}

\begin{abstract}
A method of separating heart sounds, breathing sounds, and bloodstream sounds (intended signals) from the sounds in the human body (biosignals) using microphone sensors is described as a preprocess for detecting circulatory disease such as irregular heart beat (IHB), arterial sclerosis and sleep apnea syndrome (SAS). In this paper, breathing sounds are defined as bronchial sounds. To separate intended signals from biosignals, the independent component analysis (ICA) algorithm and time-frequency masking by the expectation-maximization (EM) algorithm have been used. However, the separation filter in ICA does not work well if the recording environment has considerable reverberation. In addition, time-frequency masking of the EM algorithm is a noise and local solution problem depending on the initial value. Thus, we propose a new algorithm to solve these problems. Experimental results show that our method performs better than ICA and time-frequency masking of the EM algorithm.
\end{abstract}

Keywords: Dirichlet distribution, sounds separation, sounds in human body (biosignals), heart, breathing and bloodstream sounds (intended signals), independent component analysis (ICA), expectation-maximization (EM) algorithm

\section{Introduction}

Sounds in the human body are of great research interest [1]-[7]. In this paper, we call such sounds, consisting of heart, breathing, and bloodstream sounds, biosignals. Although most researchers have obtained only individual sound among biosignals, i.e., some researchers have separated heart sounds and noise from biosignals by using sensors attached near the heart [2], and others have separated breathing sounds and noises by using the biosignals of a human subject, in obtaining only one of these sounds by these methods is sufficient for detecting circulatory diseases, which requires judgment based on various factors. Obtaining only one of the above sounds by these methods is in adequate for the detection of circulatory diseases, which requires judgment of various factors. Therefore, we propose a method of separating three sounds, i.e., heart, breathing, and bloodstream sounds, from biosignals simultaneously. This research could contribute to the improvement of societal health. Below, we describe three benefits of our research.

First, this research could be applied to a visual-type auscultator. This auscultator could produce images from sounds (i.e., intended signals separated from biosignals), which can be used in classroom of medical and nursing science as well as in explaining the condition of patients easily.
Second, this research may be used in developing a self-monitoring system. People could evaluate their health condition by themselves in daily life using a small medical system in which our proposed method would be implemented. This device would be attached to the body surface for long periods, so conditions such as sleep apnea syndrome (SAS) might be detected. In addition, people's awareness concerning the maintenance of their health would increase much more.

Third, this research will be helpful in detecting symptoms of circulatory conditions. Of these conditions, we focus on irregular heart beat (IHB), arterial sclerosis and SAS because most people who have these diseases are not conscious of their symptoms, so many may die suddenly. IHB and arterial sclerosis cause cardiogenic embolism, which is the most dangerous disease in cerebral infarction (CI). Cardiogenic embolism is a disease in which fibrin thrombus occurring in the heart blocks off blood vessels, thus preventing blood from reaching the brain. Moreover, SAS causes abnormal pauses in breathing during sleep, and result in death during sleep. Moreover, it is said that the stress of oxidization, which is induced by SAS causes arterial sclerosis. Therefore, it is necessary that we carry out diagnosis concurrently, in an integrated manner. Thus, early detection of circulatory conditions could help prevent them. To detect these diseases, doctors generally must examine patients for signs of IHB, arterial sclerosis, and SAS using a 


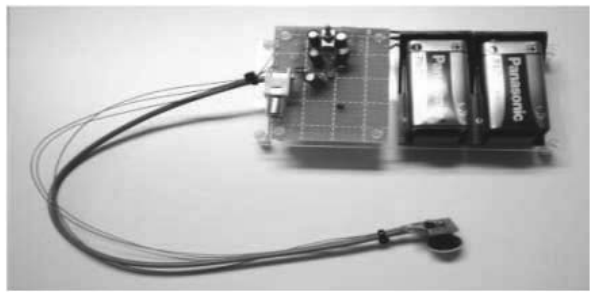

Fig.1 Microphone sensor

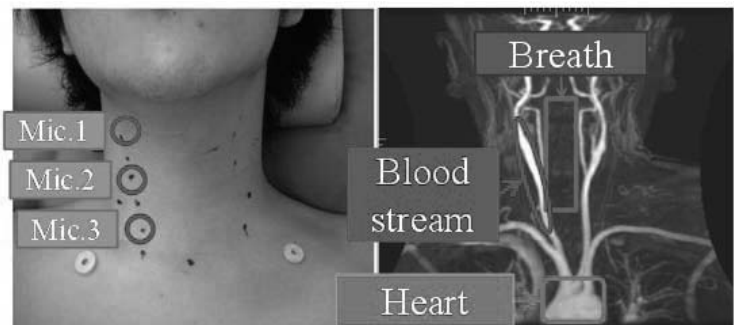

Fig.2 Placement of three microphone sensors

variety of testing equipment repeatedly. Moreover, patients must also sacrifice their much time and money. Thus, these problems may be solved by separating intended signals from biosignals and analyzing them. We aim to develop a small medical device that can detect indicators of diseases in the future. Separating intended signals from biosignals and analyzing those intended signals would be necessary for this. In this paper, we propose a method of separating intended signals from biosignals. The biosignal model can act as a blind source separation (BSS) model [6]. Independent component analysis (ICA) has often been used for sound separation [8]-[13], but it has negative points. Separation is not performed well if excessive reverberation exists in the recorded signal [9]. Time-frequency (T-F) masking [14]-[21] is widely used to separate such underdetermined mixtures. However, the expectation-maximization (EM) algorithm used in T-F masking may converge to a local solution depending on its initial value. Moreover, noise in biosignals must be reduced because it decreases the clustering accuracy of the algorithm.

Thus, we propose a new algorithm to solve these problems. The proposed method employs the 2ch-ICA to eliminate noise in the biosignals, which is robust to the diffusive noise, and then, uses the EM algorithm over the Dirichlet distribution, which resolves the local solution caused by the initial value problem, to separate the bloodstream sounds. Also, we apply a bandpass filter in separating heart and breathing sounds, leading to robustness of separation regardless of the composition of obtained biosignal data. In a subjective evaluation of experimental results, the proposed method separated intended signals from biosignals more robustly than did the existing method [7], [22].

This paper is organized as follows. Section 2 describes a biosignals model using microphone sensors. Section 3 shows existing methods (ICA and the EM algorithm) used for sound separation in the time-frequency domain. Section 4 introduces the proposed method. Section 5 reports experimental results, and Section 6 concludes this paper.

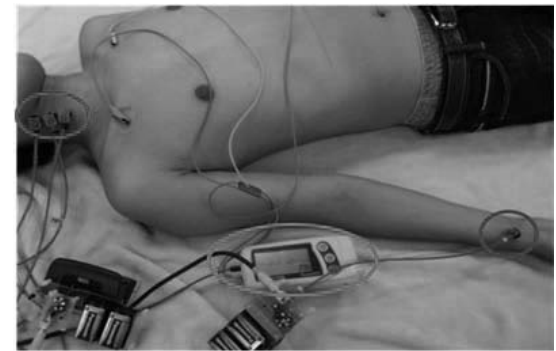

Fig.3 Actual environment of test subject (Red circle show three microphone sensors for obtaining biosignals, blue circle shows reference signal of bloodstream sounds, and green circle shows electrocardiogram (ECG))

\section{Biosignals Model}

\subsection{Biosignals with microphone sensors}

In this section, we explain the biosignals model, which can be handled as a general blind source separation (BSS). If we define $s_{k}$ as intended signals, the biosignals observed with microphone sensors are expressed as:

$$
X_{j}(t)=\sum_{k=1}^{N} \sum_{l} h_{j k}(l) s_{k}(t-l)
$$

where $s_{k}$ is the source signal, $h_{j k}(l)$ is the impulse response from source $k$ to microphone sensor $j, \quad N$ is the source number, $M$ is the number of microphone sensors, $j=1, \cdots M$, and $t$ represents time. In this work, we assume that the number of sources $N$ is known. In addition, the number of sensors $M$ is equal to $N$. We define source signals $s$ as heart, breathing, and bloodstream sounds and the observed signals $X_{j}$ as biosignals. A microphone sensor is shown in Fig.1. Placement of the microphone sensors are indicated in Fig.2. The actual environment of a test subject is shown in Fig.3.

\subsection{Short-time Fourier transform}

We transform the biosignal model to a time-frequency model by the short-time Fourier transform (STFT), because intended signals are composed of several to several dozen $\mathrm{Hz}$.

$$
X_{j}(f, \tau)=\sum_{t^{\prime}=0, t_{S}, \cdots,(L-1) t_{S}} \operatorname{win}_{\mathrm{a}}\left(t^{\prime}\right) X_{j}\left(t^{\prime}+\tau\right) e^{-i 2 \pi f t^{\prime}}
$$

Here, $\tau=\left\{0, S t_{s}, \cdots, T-1\right\}$ is the time frame index, $L$ is the sample frame, $\operatorname{win}_{\mathrm{a}}(t)$ is the Hanning window, $f_{s}$ is the sampling frequency, $f=\left\{0,(1 / L) f_{s}, \cdots,((L-1) /\right.$ L) $\left.f_{s}\right\}$ gives the frequency, and $t_{s}=1 / f_{s}$.

\subsection{Inverse short-time Fourier transform}

After we obtain separation vectors $\boldsymbol{Y}_{k}(f, \tau)$, we must transform them to the time domain from the frequency domain by inverse short-time Fourier transform (ISTFT) as follows: 


$$
Y_{k}(t)=\sum_{\tau} \operatorname{win}_{s}(t-\tau)\left[\frac{1}{L} \sum_{f} \boldsymbol{Y}_{k}(f, \tau) e^{i 2 \pi f(t-\tau)}\right]
$$

where $L$ is the sample frame, the summation over frequencies $f$ is with $f=0,(1 / L) f_{s}, \cdots((L-1) / L) f_{s}$, the summation over time frame indices $\tau$ satisfies $0 \leq t-\tau \leq(L-1) t_{s}$, and $\operatorname{win}_{s}(t-\tau)$ is the synthesis window. To achieve a perfect reconstruction, the analysis and synthesis windows should satisfy the condition

$$
\sum_{\tau} \operatorname{win}_{\mathrm{s}}(t-\tau) \operatorname{win}_{\mathrm{a}}(t-\tau)=1
$$

After this process, we obtain the intended signals.

\section{Existing Method and Problems}

\subsection{Independent component analysis}

ICA has been researched as a method of extracting target signals from mixed signals [8]-[13] and used to separate intended signals from biosignals [7]. To find the separation matrix $\mathbf{W}(f)$ for separating intended signals from biosignals,

$$
\boldsymbol{Y}(f, \tau)=\mathbf{W}(f) \boldsymbol{X}(f, \tau)
$$

is solved, where $X(f, \tau)=\left[X_{1}(f, \tau), \cdots, X_{N}(f, \tau)\right]^{\mathrm{T}}$ and $Y(f, \tau)=\left[Y(f, \tau), \cdots, Y_{N}(f, \tau)\right]^{\mathrm{T}}$. In this work, we use the information maximization approach combined with the natural gradient. The most basic and necessary preprocess is to center $\boldsymbol{X}$, i.e., subtract its mean vector $\mathbf{m}$, which is expressed as

$$
\mathbf{m}=E\{\boldsymbol{X}\}
$$

so as to make a zero-mean variable. Then, we whiten the observed vectors beforehand. A common method for whitening is to use the eigenvalue decomposition of the covariance matrix:

$$
E\left\{\boldsymbol{X} \boldsymbol{X}^{\mathrm{T}}\right\}==\mathbf{E D E}^{\mathrm{T}}
$$

where $\mathbf{E}=\left[\mathbf{e}_{1} \cdots \mathbf{e}_{N}\right]$ is the orthogonal matrix of eigenvectors of $E\left\{\boldsymbol{X} \boldsymbol{X}^{\mathrm{T}}\right\}, \mathbf{D}$ is the diagonal matrix of its eigenvalues, and $\mathbf{D}=\operatorname{diag}\left(d_{1}, \ldots, d n\right)$. Therefore, whitening can now be accomplished by

$$
\tilde{\mathbf{x}}=\mathbf{E D}^{-\frac{1}{2}} \mathbf{E}^{\mathrm{T}} \boldsymbol{X}
$$

where the matrix $\mathbf{D}^{-\frac{1}{2}}$ is computed by a simple component-wise operation as $\mathbf{D}^{-\frac{1}{2}}=\operatorname{diag}\left(d_{1}^{-\frac{1}{2}} \cdots d_{n}^{-\frac{1}{2}}\right)$. These steps are important for ICA because the output of ICA adheres to the signal subspace that is identified by the $N$ eigenvectors, $\mathbf{e}_{1} \cdots \mathbf{e}_{N}$. This means that the following ICA algorithm is not solved in the noise subspace, which consequently stabilizes the algorithm and also reduces noise. Next, we perform signal separation by using the complex-valued inverse of the mixing matrix $\mathbf{W}(f)$. This matrix is $N \times M$, and its elements are $W_{i j}(f)$. The optimal $\mathbf{W}(f)$ is obtained by

$$
\begin{aligned}
\Delta \mathbf{W}(f) & =\eta\left[\boldsymbol{I}-\left\langle\varphi(\boldsymbol{Y}(f, \tau)) \boldsymbol{Y}^{H}(f, \tau)\right\rangle_{t}\right] \mathbf{W}(f) \\
& +\mathbf{W}(f)
\end{aligned}
$$$$
\varphi\left(\boldsymbol{Y}_{\boldsymbol{k}}\right)=\tanh \left[\operatorname{real}\left(\boldsymbol{Y}_{\boldsymbol{k}}\right)\right]+j \cdot \tanh \left[\operatorname{imag}\left(\boldsymbol{Y}_{\boldsymbol{k}}\right)\right]
$$

where $\eta$ is a step parameter, $\langle\cdot\rangle_{t}$ denotes the averaging operator over time, and $\varphi(\cdot)$ is an element-wise nonlinear function for a complex signal $\boldsymbol{Y}_{k}, \boldsymbol{Y}^{H}$ is the complex conjugate of $\boldsymbol{Y}_{\boldsymbol{k}}$, and $\operatorname{real}\left(\boldsymbol{Y}_{\boldsymbol{k}}\right)$ and $\operatorname{imag}\left(\boldsymbol{Y}_{\boldsymbol{k}}\right)$ are the real and imaginary parts of $\boldsymbol{Y}_{k}$, respectively. However, the separation matrix still has scaling and permutation ambiguity.

With regard to the scaling ambiguity, obtaining only a delayed version of $s_{k}(t)$ at output $y_{k}(t)$ is desirable. However, achieving this by ICA is difficult, so we apply the minimal distortion principle [23]. Let $\mathbf{H}(f)$ be the unknown mixing matrix. The diagonal matrix $\mathbf{A}(f)$ should satisfy

$$
\mathbf{A}(f) \mathbf{W}(f) \mathbf{H}(f)=\operatorname{diag}[\mathbf{H}(f)]
$$

If ICA works well, $\mathbf{W}(f) \mathbf{H}(f)=\mathbf{D}(f)$ have equality. $\mathbf{D}(f)$ is a diagonal matrix. Thus, we can estimate $\mathbf{H}(f)$ by

$$
\mathbf{H}(f)=\mathbf{W}^{-1}(f) \mathbf{D}(f)
$$

As a result, we obtain a new $\mathbf{W}(f)$ that solves the scaling ambiguity by

$$
\mathbf{W}(f) \leftarrow \operatorname{diag} \mathbf{W}^{-1}(f) \cdot \mathbf{W}(f)
$$

The permutation ambiguity of ICA in each frequency bin should be aligned so that a separated signal in the time domain contains frequency components of the same source signal. Therefore, we must find permutation matrix $\mathbf{P}(f)$ that satisfies as follows:

$$
\mathbf{W}(f) \leftarrow \mathbf{P}(f) \mathbf{W}(f)
$$

In this work, we use the permutation solving method in [24] to find permutation matrix $\mathbf{P}(f)$. Thus, separated filters $\mathbf{W}(f)$ are generated.

\subsection{Time-frequency masking with EM algorithm}

Heart, breathing and bloodstream sounds are independent in the human body, so the main components of biosignals are made up of a mixture of them. Thus, we can obtain them if we can classify their borderline. Therefore, we use the EM algorithm [22] to identify the borderline. This algorithm yields estimates of the parameters in the set and posterior probabilities for all times. We normalize $\boldsymbol{X}(f, \tau)$ as

$$
X(f, \tau) \leftarrow \frac{X(f, \tau)}{\|X(f, \tau)\|}
$$

to eliminate the effect of the source amplitude. To model vectors for each source, we follow the idea described in [18].

E-step: $\gamma\left(\boldsymbol{X}(f, \tau) \mid \boldsymbol{\mu}_{k}, \sigma_{k}\right)$

$=\frac{1}{\left(\pi \sigma_{k}^{2}\right)^{M-1}} \exp \left(\frac{-\left\|\boldsymbol{X}(f, \tau)-\left(\boldsymbol{\mu}_{k}^{H} \boldsymbol{X}(f, \tau) \boldsymbol{\mu}_{k}\right)\right\|^{2}}{\sigma_{k}^{2}}\right)$

Here, $\sigma_{k}^{2}$ is the variance and $\boldsymbol{\mu}_{k}$ is the mean vector. Then, the density function for the observed signals can be given by using Eq.(16) in

$$
\gamma(X(f, \tau) \mid \theta)=\sum_{k=1}^{N} \varepsilon_{k} \gamma\left(X(f, \tau) \mid \boldsymbol{\mu}_{k}, \sigma_{k}\right)
$$

where the mixture ratio $\varepsilon$ is $0<\varepsilon_{k}<1, \varepsilon_{1}+\cdots+\varepsilon_{k}=1$, and the parameter $\theta=\left\{\boldsymbol{\mu}_{k}, \sigma_{k}, \varepsilon_{k}\right\}$. By introducing the posterior probability, 


$$
P\left(C_{k} \mid \boldsymbol{X}(f, \tau), \theta^{t}\right)=\frac{\varepsilon_{k} \gamma\left(\boldsymbol{X}(f, \tau) \mid \boldsymbol{\mu}_{k}, \sigma_{k}\right)}{\gamma(\boldsymbol{X}(f, \tau) \mid \theta)}
$$

M-step:

$$
\begin{aligned}
& Q\left(\theta \mid \theta^{t}\right) \\
& =\sum_{t}^{T} \sum_{k=1}^{N} P\left(C_{k} \mid X(f, \tau), \theta^{t}\right) \log \varepsilon_{k} \gamma\left(X(f, \tau) \mid \boldsymbol{\mu}_{k}, \sigma_{k}\right)
\end{aligned}
$$

where $Q$ is the cost function. We must maximize this in the EM algorithm. With regard to $\boldsymbol{\mu}_{k}$, we use the unit norm $\left\|\boldsymbol{\mu}_{k}\right\|^{2}=1$, a Lagrange multiplier $\lambda$, and the derivative. We derive the following updated expression:

$$
\mathrm{B}_{k}=\sum_{\tau}^{T} P\left(C_{k} \mid \boldsymbol{X}(f, \tau), \theta^{t}\right) \boldsymbol{X}(f, \tau) \boldsymbol{X}^{H}(f, \tau) .
$$

In Eq.(20), the optimal $\boldsymbol{\mu}_{k}$ is given by the eigenvector corresponding to the maximum eigenvalue.

$$
\begin{gathered}
\sigma_{k}^{2}=\frac{\sum_{\tau}^{T} P\left(C_{k} \mid X(f, \tau), \theta^{t}\right)|| \boldsymbol{X}(f, \tau)-\left(\boldsymbol{\mu}_{k}^{H} \boldsymbol{X}(f, \tau) \boldsymbol{\mu}_{k}\right) \|^{2}}{(M-1) \cdot P\left(C_{k} \mid X(f, \tau), \theta^{t}\right)} \\
\varepsilon_{k}=(1 / T) \sum_{\tau}^{T} P\left(C_{k} \mid X(f, \tau), \theta^{t}\right)
\end{gathered}
$$

Both Eq.(21) and (22) are also updating expressions, and they are repeated until convergence. We solve the permutation problem using the method in [24]. The observed signals $X(f, \tau)$ should belong to class $C_{k}$ when the source $s_{k}$ is the most dominant in $X(f, \tau)$. Hence we make a time-frequency mask for the source separation performance through

$\boldsymbol{Y}_{k}(f, \tau)= \begin{cases}X_{J}(f, \tau), & \text { if } P\left(C_{k} \mid \boldsymbol{X}\right) \geq P\left(C_{k^{\prime}} \mid \boldsymbol{X}\right), k^{\prime} \neq k \\ 0, & \text { otherwise }\end{cases}$

where $J$ is the index of an arbitrarily selected reference microphone that is used to construct the separated signals. At the end of the flow, time-domain output signals $Y_{k}(t)$ are calculated with an inverse STFT to the frequency-domain separated signals $\boldsymbol{Y}_{k}(f, \tau)$.

\subsection{Problems of existing methods}

\subsubsection{ICA problem}

As previously mentioned, existing methods have demerits. The ICA algorithm has reverberation problems. It works well if the intended sound and noise are expressed as point sources. However, such an environment is comparatively rare in the real world. In reality, diffusive noises from difference directions are the main components, so the ICA algorithm does not work well in this case. In the biosignal model, noise is not a point source but naturally diffusive. Therefore, we changed the ICA noise reduction method, not its separation method. In this paper, the algorithm is called 2-ch ICA because it separates biosignals from two sound sources, thus two channels, using the primary and reference microphone sensors. The detail information of this algorithm is introduced in section 4.2.

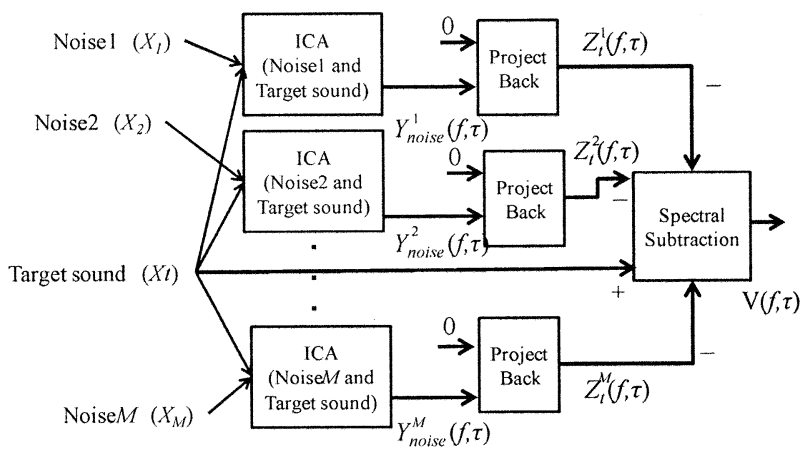

Fig.4 2-ch ICA in biosignal model

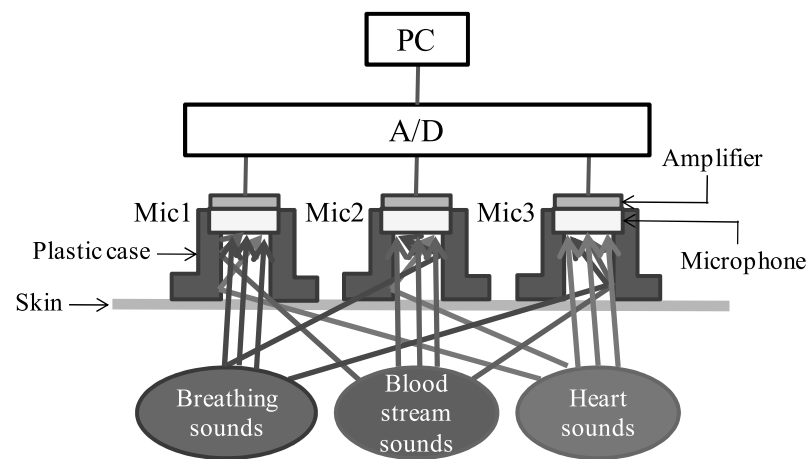

Fig.5 Recording of biosignals by three microphone sensors (after recording, biosignals pass through the $\mathrm{AD}$ converter and are processed by PC)

\subsubsection{EM algorithm problems}

To remove noise, a bilateral filter was used in [22], but necessary components for the separation may have been removed because the filter is not designed to handle absolute signal information. On the other hand, the accuracy of noise reduction in 2-ch ICA is better than when using bilateral filter because 2-ch ICA removes noise using each signal information.

Moreover, the EM algorithm may depend on the initial value. This algorithm repeatedly adjusts parameters (mean $\boldsymbol{\mu}_{k}$, variance $\sigma_{k}^{2}$ and mixture ratio $\varepsilon_{k}$ ) until convergence, so the results of this algorithm may fall into a local solution if we do not set the optimal initial value. For example, if we do not know the number of sources, we must prepare for sufficient (i.e., more than the number of sensors) normal distribution and we set the initial parameters for Gaussian mixture models (GMM). However, if we apply the GMM directly, the algorithm may form a cluster using multiple normal distributions. Consequently, this algorithm falls into a local solution. Thus, we cannot obtain optimal results of the EM algorithm. Therefore, we introduce the Dirichlet distribution [25] to the EM algorithm for this problem.

\subsubsection{Robustness of separating heart and breathing sounds}

There is a problem of robustness in obtaining heart and breathing sounds from biosignals. In this paper, we focus on IHB and SAS. IHB is a condition in which heart rate 
and rhythms are irregular. In SAS, breathing stops or is infrequent. Therefore, to detect IHB, we must detect a heartbeat similar to the separation results of heart sounds. Moreover, to detect SAS, we must distinguish inhalation and exhalation from the separation results of breathing sounds. However, the existing methods [7], [22] do not work well if biosignals do not contain strong components of heart or breathing sounds, e.g., biosignals from elderly people. That is, separation results lack robustness in such cases. Therefore, we apply a bandpass filter to solve this problem.

\section{Proposed Method}

\subsection{Bandpass filter}

The frequency band of heart sounds is from 20 to $100 \mathrm{~Hz}$ [6], which that of breathing sounds is from 25 to $800 \mathrm{~Hz}$ [5]. Therefore, in this study, we designed the cut off frequencies of the bandpass filter not to overlap the frequency bands, i.e. from 30 to $50 \mathrm{~Hz}$ for heart sound, and from 300 to $700 \mathrm{~Hz}$ for breath sounds. It is clear that the bandpass filter for the heart sounds also extracts the breathing sounds, but the heart sounds become dominant. Thus, we could obtain heart and breathing sounds by this method.

\subsection{2-ch ICA to reduce noise}

ICA has been researched as a method of extracting target signals from mixed signals. The BSS method of ICA [4]-[9] shows good performance in a situation where the target sound and noise are composed as a point source of sound, such as in a voice conversation. However, ICA does not perform well in a situation with considerable diffusive noise [9]. If we use simple ICA, we cannot obtain the intended signals because the biosignal model is dominated by diffusive noise. Thus, we use 2-ch ICA to solve this problem. For example, if we define biosignals of Mic. 2 as the target sound, 2-ch ICA reduces the noise of Mic. 1 and Mic. 3 from the biosignals of Mic. 2.

First, we carry out 2 ch-ICA with a primary microphone sensor including more the target sound and a reference microphone including more the noise, i.e., ICA of noise1 and the target sound, as in Fig.4.

Next, we separate a sound in which noise components are dominant, i.e., $\mathrm{Y}_{\text {noise }}^{1}(f, \tau)$, as in Fig.4, and a sound in which target components are dominant by 2 ch-ICA and cancel the sound containing the target components. Finally, we subtract the sound containing many noise components from the signal observed with the primary microphone, and thus reduce the noise.

The mathematical derivations of $2 \mathrm{ch}-\mathrm{ICA}$ are shown next. We designate the observed signal vector in the time-frequency domain as

$$
\boldsymbol{X}_{m}(f, \tau)=\left[X_{t}(f, \tau), X_{m}(f, \tau)\right]^{\mathrm{T}}
$$

where $X_{m}(f, \tau)$ is the observed signal of reference microphone $m$ and $X_{t}(f, \tau)$ is the target microphone.

$$
\boldsymbol{Y}_{m}(f, \tau)=\mathbf{W}_{m}(f) \boldsymbol{X}_{m}(f, \tau)
$$

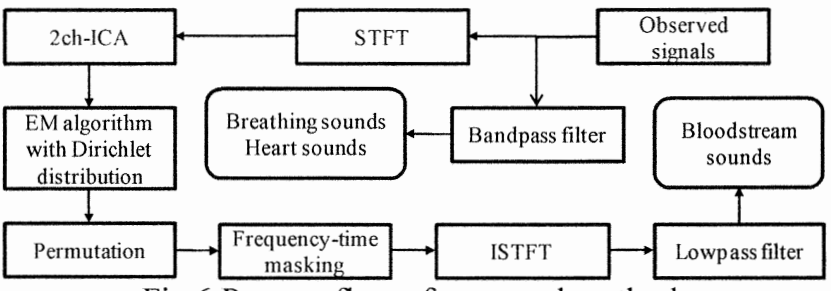

Fig.6 Process flow of proposed method

whose elements are $W_{k j}(f)$. This matrix is a complex-valued unmixed matrix. To obtain the optimal $\mathbf{W}_{m}(f)$, the updated Eqs. (9) and (10) are repeated until convergence. In this method, we are only required to estimate a noise component. Thus, the $Y_{t}^{(m)}(f, \tau)$ including more target signal components is set to zero from the output signal vector $\boldsymbol{Y}_{m}(f, \tau)$.

$$
\boldsymbol{U}_{m}(f, \tau)=\left[\frac{0}{Y_{\text {noise }}^{(m)}}\right]
$$

Then, we apply the backprojection [26] method to remove the ambiguity of the amplitude. This procedure yields

$$
\boldsymbol{Z}_{m}(f, \tau)=\mathbf{M}_{m}^{+}(f) \boldsymbol{U}_{m}(f, \tau)
$$

where $\mathbf{M}_{m}^{+}(f)$ is the Moore-Penrose pseudo-inverse matrix of $\mathbf{W}_{m}(f), \boldsymbol{Z}_{m}(f, \tau)=\left[Z_{t}^{(m)}(f, \tau), Z_{r}^{(m)}(f, \tau)\right]^{\mathrm{T}}$ is the $m$-th estimated noise vector and each of the components are the $m$-th estimated noise input to a primary microphone and a reference microphone, respectively. Finally, we use aberrant spectral subtraction as follows:

$|V(f, \tau)|=$

$$
\left\{\begin{array}{c}
\left\{\left|X^{(t)}(f, \tau)\right|^{2}-\sum_{j=1}^{M} \alpha_{j}\left|Z_{t}^{(m)}(f, \tau)\right|^{2}\right\}^{1 / 2} \\
\left(i f\left|X^{(t)}(f, \tau)\right|^{2}-\sum_{j=1}^{M} \alpha_{j}\left|Z_{t}^{(m)}(f, \tau)\right|^{2} \geq 0\right) \\
\left.\delta|| X^{(t)}(f, \tau)\right|^{2} \mid \quad(\text { otherwise }) \\
V(f, \tau)=|V(f, \tau)| e^{-j a r g\left(x_{t}(f, \tau)\right)}
\end{array}\right.
$$

where $V(f, \tau)$ is the output signal, $\alpha_{j}$ is the oversubtraction parameter, $\sum_{j}^{M} \alpha_{j} \cong 1$, and $\delta$ is the flooring parameter.

A diagram of recording biosignals using multiple microphone sensors is shown in Fig.5. In cases like this, Mic. 1 observes biosignals which are with a high proportion of breathing components. If we define biosignals of Mic. 2 as the target sound, this process eliminates breathing and heart components in Mic. 1 and Mic. 3 from biosignals of Mic. 2.

\subsection{Robust EM algorithm}

\subsubsection{Dirichlet distribution}

We describe the Dirichlet distribution briefly in this section. By inspecting the form of the multinomial distribution [25], we see that the conjugate prior is given by

$$
p(\varepsilon \mid \omega) \propto \prod_{k=1}^{N} \varepsilon_{k}^{\omega-1}
$$

where the separation matrix $\mathbf{W}_{m}(f)$ is the $N \times M$ matrix 
Biosignals (observed signals)

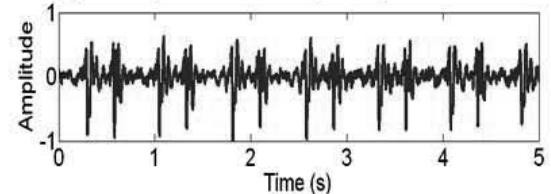

(a)

ICA

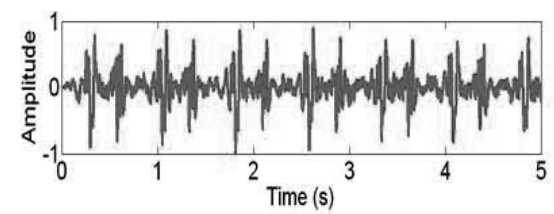

(d)

EM algorithm

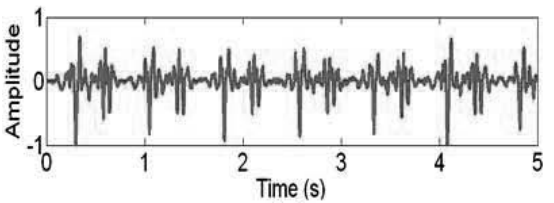

(g)

Proposed method

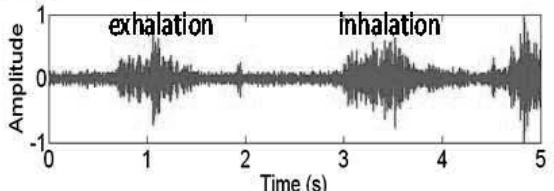

(j)

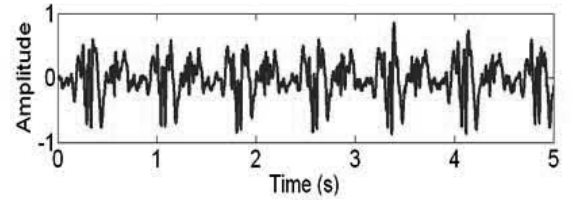

(b)

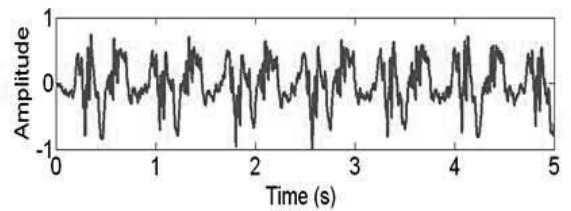

(e)

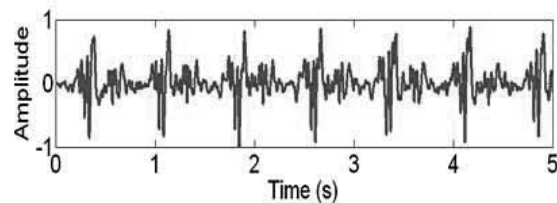

(h)

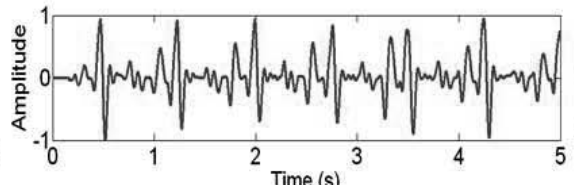

(k)

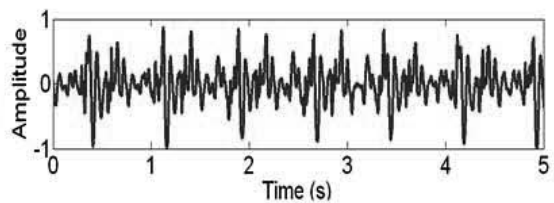

(c)

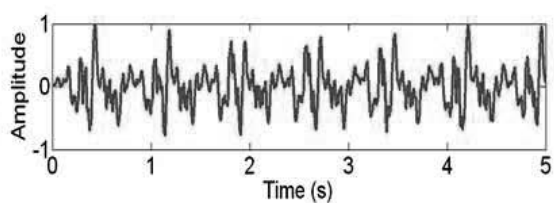

(f)

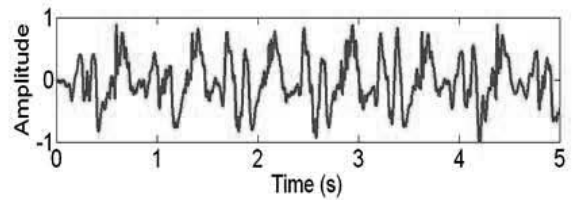

(i)

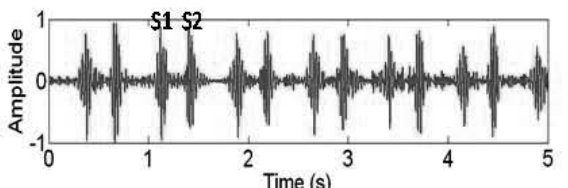

(1)

Fig.7 Experimental results for one human subject in their twenties (observed signals at (a) Mic. 1, (b) Mic. 2, and (c) Mic. 3: results using ICA [7], (d) breathing sounds, (e) bloodstream sounds, and (f) heart sounds: results of EM algorithm [22], (g) breathing sounds, (h) bloodstream sounds, and (i) heart sounds: results of proposed algorithm, (j) breathing sounds, (k) bloodstream sounds, and (1) heart sounds)

Biosignals (observed signals)

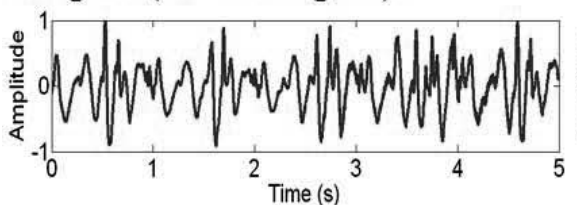

(a)

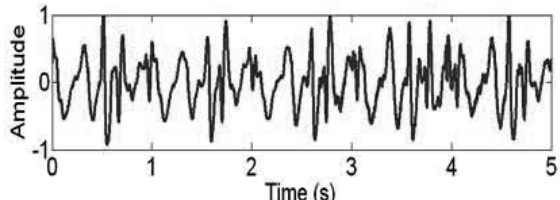

(b)

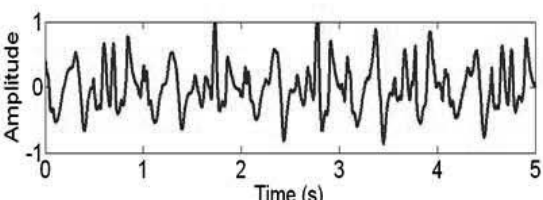

(c)

ica

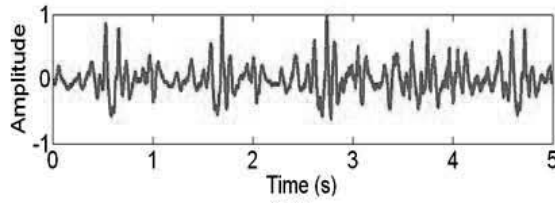

(d)

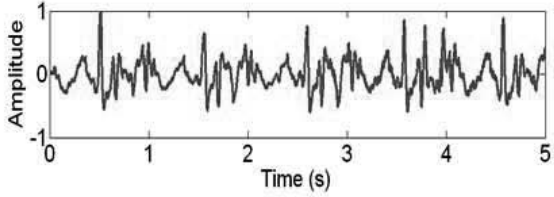

(e)

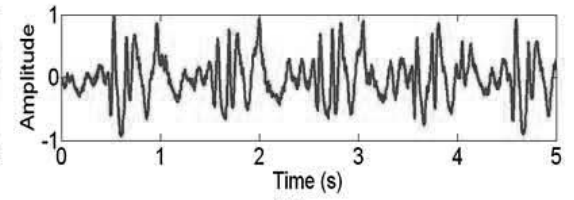

(f)

EM algorithm

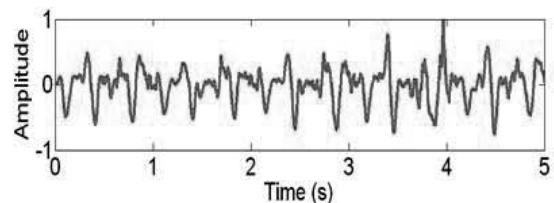

(g)

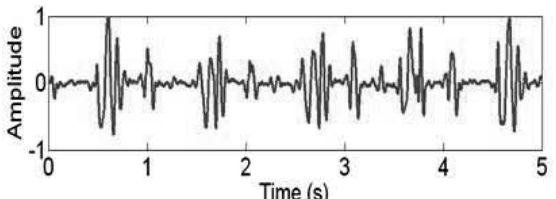

(h)

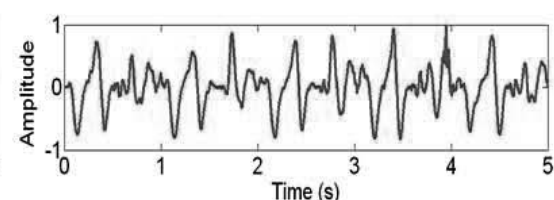

(i) 
Proposed method

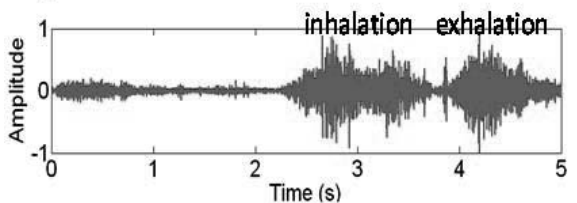

(j)

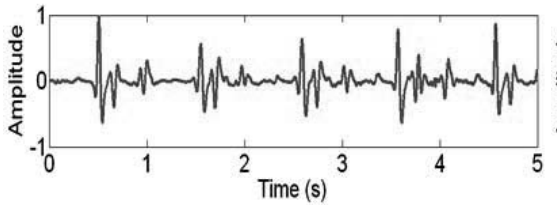

$(\mathrm{k})$

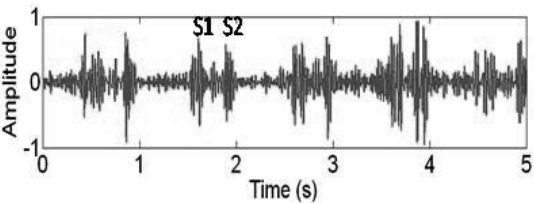

(1)

Fig.8 Experimental results for another human subject in their twenties (observed signals at (a) Mic. 1, (b) Mic. 2, and (c) Mic. 3: results using ICA [7], (d) breathing sounds, (e) bloodstream sounds, and (f) heart sounds: results of EM algorithm [22], (g) breathing sounds, (h) bloodstream sounds, and (i) heart sounds: results of proposed algorithm, (j) breathing sounds, (k) bloodstream sounds, and (l) heart sounds)

Table 1 Experimental conditions

\begin{tabular}{|l|l|}
\hline Number of microphone sensors & $M=3$ \\
\hline Number of sources & $N=3$ \\
\hline STFT frame size & 512 points \\
\hline STFT shift size & 64 points \\
\hline Sampling rate & $f_{s}=4 \mathrm{kHz}$ \\
\hline Length of observed signals & $5 \mathrm{~s}$ \\
\hline Step-size parameter & 0.001 \\
\hline Over-subtraction parameter & 0.5 \\
\hline Flooring parameter & 0.1 \\
\hline Hyper-parameter & 0.98 \\
\hline
\end{tabular}

where $0 \leq \varepsilon_{k} \leq 1, \varepsilon_{1}+\cdots+\varepsilon_{N}=1$ and $\omega$ is a hyperparameter. $\boldsymbol{\omega}$ denotes $\left(\omega_{1} \cdots \omega_{N}\right)^{T}$. If we set the hyperparameter to be smaller than 1 , a small minority of mixture ratio $\varepsilon_{k}$ becomes a large number and the others become a near-zero number. The Dirichlet distribution is normalized by

$$
\operatorname{Dir}(\boldsymbol{\varepsilon} \mid \boldsymbol{\omega})=\frac{\Gamma\left(\omega_{0}\right)}{\Gamma\left(\omega_{1}\right) \cdots \Gamma\left(\omega_{N}\right)} \prod_{k=1}^{N} \varepsilon_{k}^{\omega-1}
$$

where $\Gamma(\cdot)$ is the gamma function and $\omega_{0}=\sum_{k=1}^{N} \omega_{k}$.

\subsubsection{EM algorithm with Dirichlet distribution}

In this section, we propose the EM algorithm with the Dirichlet distribution. We apply the Dirichlet distribution to the biosignals model as a prior distribution of the mixture ratio as follows:

$$
p\left(\varepsilon_{1} \cdots \varepsilon_{N}\right)=\frac{\Gamma(N \cdot \omega)}{\Gamma(\omega)^{N}} \prod_{k=1}^{N} \varepsilon_{k}^{\omega-1} .
$$

Next, we apply GMM to the data after 2ch-ICA. A cost function with power-weighted average likelihood is represented by

$$
L(\theta)=Q\left(\theta \mid \theta^{t}\right)+\log p(\theta)
$$

Generally, the likelihood maximization causes overadaptation if the number of training samples is relatively low; thus, the term $\log p(\theta)$ is applied to control overadaptation. $\theta^{t}$ shows a parameter obtained at $t$-th. We consider the prior Eq.(32) for the mixture ratio $\varepsilon_{k}$, but no prior for the Gaussian parameters $\boldsymbol{\mu}_{k}$ and $\sigma_{k}$. Thus, we obtain

$$
\log p(\theta)=(\omega-1) \sum_{k=1}^{N} \log \varepsilon_{k}+\text { const }
$$

The property of mixture ratio $\sum_{k=1}^{N} \varepsilon_{k}=1$ should be satisfied. Thus, we obtain

$$
L_{\varepsilon}\left(\varepsilon_{k}, \lambda\right)=Q\left(\theta \mid \theta^{t}\right)+\log p(\theta)+\lambda\left(\sum_{k=1}^{N} \varepsilon_{k}-1\right)
$$

with the Lagrange multiplier $\lambda$. After we calculate the derivative of $L_{\varepsilon}\left(\varepsilon_{k}, \lambda\right)$ with respect to $\varepsilon_{k}$ for $k=$ $\{1, \cdots, N\}$, we obtain

$$
\sum_{\tau}^{T} P\left(C_{k} \mid \boldsymbol{X}(f, \tau), \theta^{t}\right)+(\omega-1)+\pi_{k} \lambda=0
$$

Summing these up with $k=\{1, \cdots, N\}$, we have

$$
\lambda=-\{T+N(\omega-1)\}
$$

Then, mixture ratio $\varepsilon_{k}$ with MAP estimation is updated to

$$
\varepsilon_{k}=\frac{\sum_{\tau}^{T} P\left(C_{k} \mid X(f, \tau), \theta^{\prime}\right)+(\omega-1)}{T+N \cdot(\omega-1)}
$$

We then substitute Eq.(38) for Eq. (22). In this way, the EM algorithm with the Dirichlet distribution is more robust than only the EM algorithm with respect to initial-value dependence. In this experiment, we assumed that the information on the number $N$ of sources was given in priori. For such a case, it is advantageous to choose a large number for the hyperparameter $\omega$ in Eq.(32) so that each cluster has almost the same weight $\varepsilon$ based on Eq. (38). The whole process flow of the proposed method is shown in Fig.6.

\section{Experimental Results}

\subsection{Sensor system and recording condition}

In this experiment, we attached three MX-E4758 microphone sensors (Primo Co., Ltd.) to the right common carotid artery (as in Figs.2 and 3) and recorded $5 \mathrm{~s}$ of biosignals for each of ten human subjects in their twenties and five human subjects in their seventies. We can obtain the biosignals, including the intended signals, simultaneously in this way. The proposed method was coded in MATLAB to show the performance. The experimental conditions are shown in Table 1. Also, other parameters are initially set as $\sigma^{2}=0.1, \varepsilon_{k}=1 / N$ and $\mathbf{a}_{k} \leftarrow \boldsymbol{X}_{k}(f, \tau)$.

\subsection{Evaluation measure and separation results}

To check if the separated signals (Figs.7(j), (k), (l)) are the intended signals, we prepared reference signals for each 
of the intended signals and checked the waveforms and pitch because the original breathing sounds, bloodstream sounds, and heart sounds cannot be obtained individually. Therefore, the separation result cannot be expressed as a signal-to-noise ratio or signal-to-interference ratio. We used the sounds in a radial artery at the wrist of a human subject for bloodstream sounds because these sounds are far from the heart and trachea and are generated in almost all bloodstream components. For heart sounds, we used an electrocardiogram (ECG). These reference signals were obtained from the same human subject. For breathing sounds, we checked for two distinct sections in the waveform that represent inhalation and exhalation. These evaluation measures were recommended by doctors at the Shiga University of Medical Science. Therefore, they have sufficient meaning as evaluation measures. Fig. 7 shows a result for one human subject in their twenties and Fig. 8 shows another result for another human subject in their twenties.

Here, we must examine the computation complexity because the proposed method integrates BPF, STFT, 2ch-ICA, and EM algorithm with the Dirichlet distribution. It is obvious that realizing the algorithm in real-time application is highly difficult. However, the target application of this research does not require the real-time processing to identify any the abnormality in long-time analysis. For diseases with gradual changes such as IHB, arterial sclerosis, and SAS, diagnosis by long-time analysis of biosignals in daily life is important, which means we do not require real-time performances for this application.

\subsubsection{Breathing sounds}

Breathing sounds appear as two clusters if the separation filters work well because breathing has two actions, i.e., inhalation and exhalation (Fig.9). That is, each breathing sound signal corresponds to inhalation and exhalation. As mentioned in section 4.1, ICA and the EM algorithm do not work well for biosignals that do not have many breathing sound components (Figs.7(d) and (g)) because two distinct clusters are not visible. However, we obtained two clusters indicating inhalation and exhalation by using a bandpass filter (Fig.7(j)). Hence, it is clear that the result obtained by the proposed method (Fig.7(j)) represents breathing sounds. We believe that sleep apnea syndrome can be detected early by observing these clusters in sound signals obtain from people wearing a device implementing this proposed method while they sleep.

\subsubsection{Heart sounds}

In the results of ICA (Fig.7(f)) and the EM algorithm (Fig.7(i)), S1 and S2 are not clear because they are buried in noise. This is thought to be due to the severe reverberation problem in ICA and the initial-value dependence and noise in the EM algorithm. However, S1 and S2 were clear when the bandpass filter was used (Fig.7(1)). Moreover, it shows that the periodicities of heart sounds and ECG are in agreement because S1 corresponds to the S-wave (Fig.10)

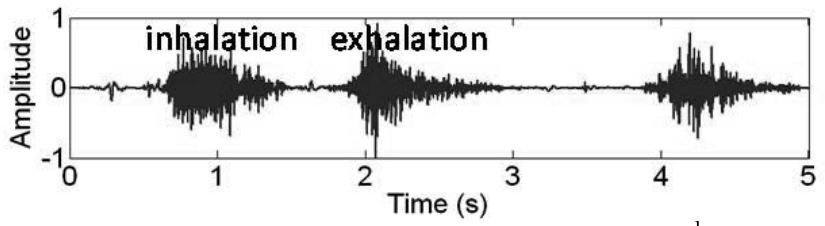

Fig.9 Reference waveform of breathing sounds ${ }^{1}$ [27]

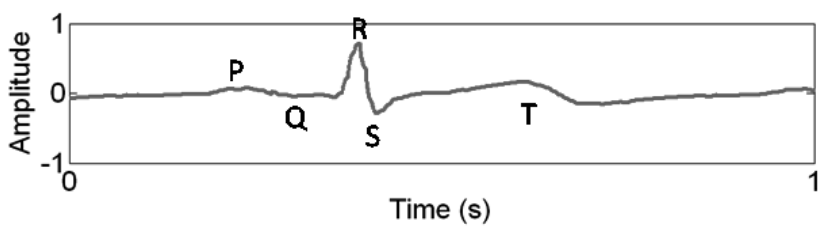

Fig.10 1-s waveform of ECG

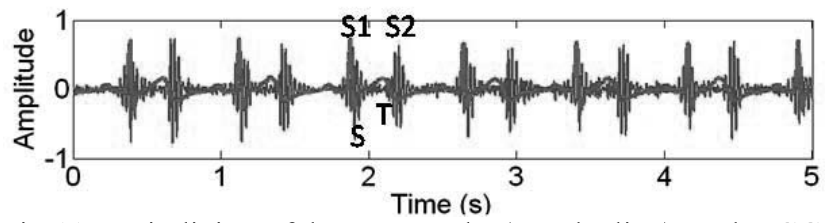

Fig.11 Periodicity of heart sounds (purple line) and ECG (red line)

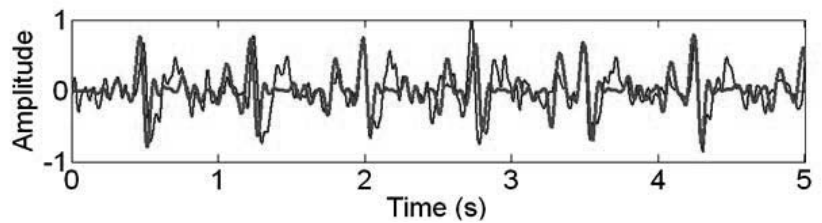

Fig.12 Periodicity and shape of bloodstream sounds (green line) and reference waveform of bloodstream sounds, i.e., arteria radialis sounds (blue line)

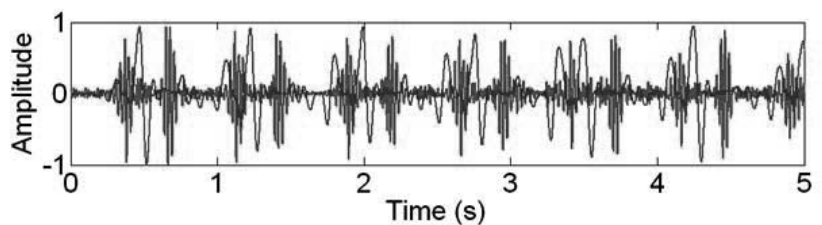

Fig.13 Relationship between bloodstream sounds (green line) and heart sounds (purple line)

and S2 corresponds to the T-wave (Fig.10) of the ECG in Fig.11. Hence, it is clear that the result obtained by the proposed method (Fig.7(1)) represents heart sounds. S1 and S2 do not appear if a patient has an abnormal cardiac rhythm. That is, we can detect an abnormal cardiac rhythm early by checking the periodicities of $\mathrm{S} 1$ and $\mathrm{S} 2$.

\subsubsection{Bloodstream sounds}

The shape of the result obtained by the proposed method (Fig.7(k)) is more similar to the reference blood stream sounds (blue line in Fig.12) than those obtained by ICA (Fig.7(e)) and by the EM algorithm (Fig.7(h)). Moreover, we can verify that the periodicities of bloodstream sounds and the reference sound in Fig. 12 match. Also, the result obtained by the proposed method (Fig.7(k)) appears 

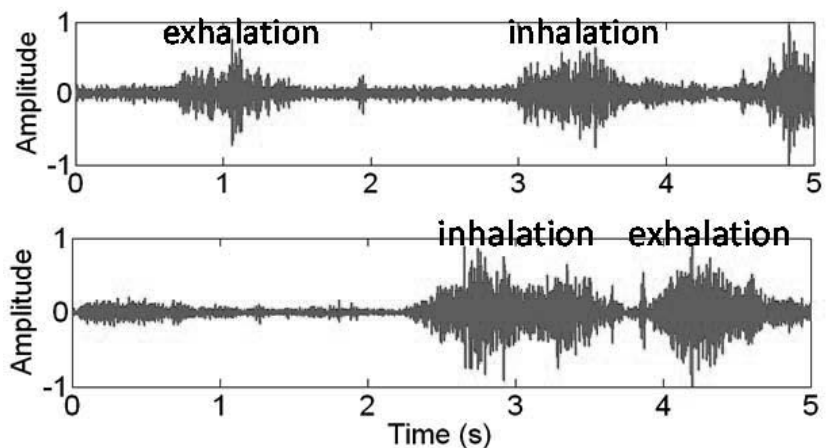

Fig.14 (above) the resultant breathing sounds for one human subject in their twenties and (below) the resultant breathing sounds for another human subject in their twenties
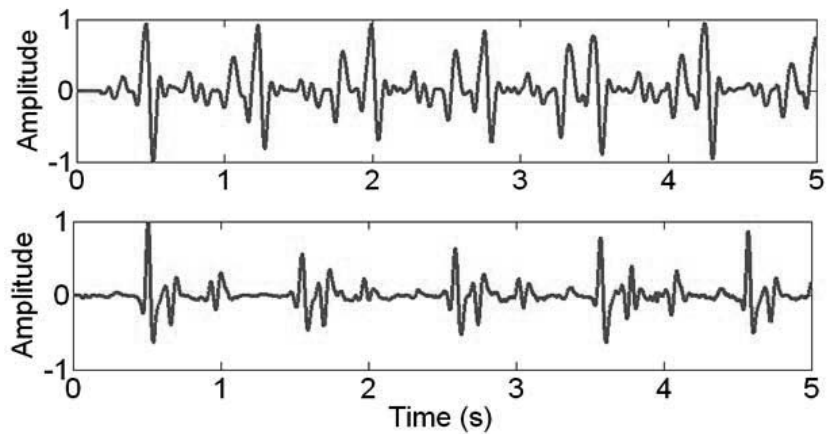

Fig. 15 (above) the resultant bloodstream sounds for one human subject in their twenties and (below) the resultant bloodstream sounds for another human subject in their twenties
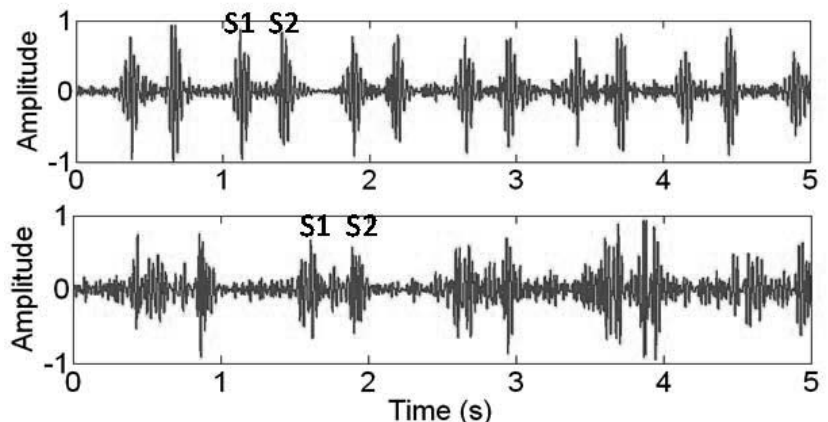

Fig.16 (above) the resultant heart sounds for one human subject in their twenties and (below) the resultant heart sounds for another human subject in their twenties

between $\mathrm{S} 1$ and $\mathrm{S} 2$ in Fig.13. These indicate that the proposed method is effective because of the combination of 2ch-ICA and the EM algorithm with Dirichlet distribution. Consequently, it is clear that the result obtained by the proposed method (Fig.7(k)) represents bloodstream sounds.

\subsubsection{Comparing for different human subjects}

The resultant breathing, bloodstream and heart sounds in Fig.14, Fig.15 and Fig.16, respectively, where processed by the same proposed method for different human subjects. As

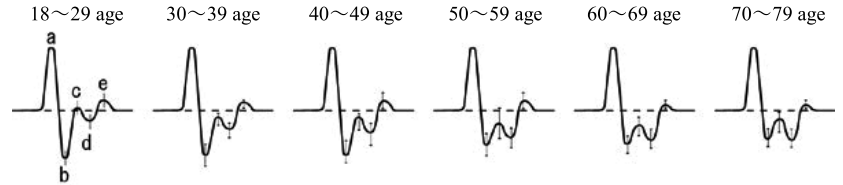

Fig.17 Second derivations of photoplethysmogram ${ }^{2}$ [28].

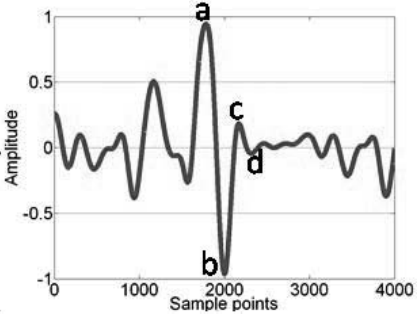

(a)

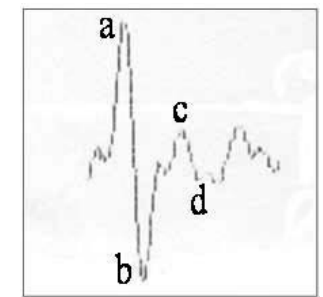

(b)
Fig.18 (a) 1-s waveform of resultant bloodstream sounds of human subject in their twenties from Fig.7(k), (b) SDPTG using pulse wave sensor machine for same human subject

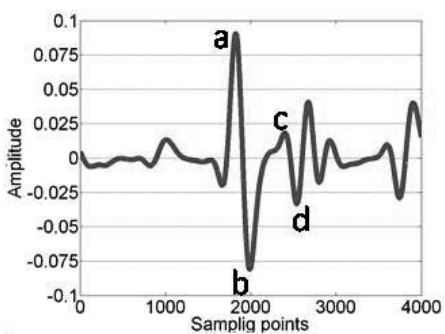

Fig.19 1-s waveform of resultant blood stream sounds of another human subject in their twenties

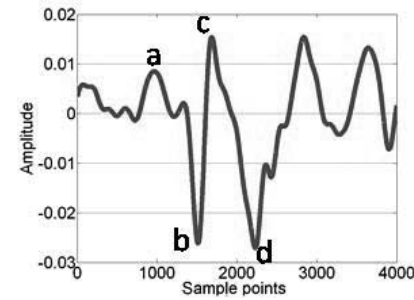

(a)

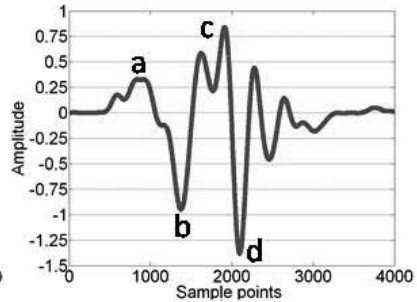

(b)
Fig.20 (a), (b) 1-s waveform of resultant bloodstream sounds of two human subjects in their seventies

you can see, with regard to Fig.14, it is found that both waveforms show two actions, i.e., inhalation and exhalation. Also, it can be seen that the waveforms are almost the same and have the a - $d$ peaks marked in Figs.18(a) and 19. It is further evident from Fig. 16 that both waveforms have S1 and $\mathrm{S} 2$. In addition, we can determine the heart rate by counting S1 and S2. We can see that the interval between breaths and the number of S1 and S2 are different between the two waveforms in Figs.14 and 16. However, this means not the degradation of separation accuracy but an individual difference in breathing and heartbeat. Therefore, the effectiveness of the proposed algorithm was clearly shown. 


\subsubsection{Second derivations of photoplethysmogram}

We show the waveform (Fig.17) of second derivations of photoplethysmogram (SDPTG). From the SDPTG, doctors judge the hardness of blood vessels and predict arterial sclerosis by analyzing the fraction of the a-wave and each wave in Fig.17. Moreover, the patients might present hardness of blood vessels and arterial sclerosis if the line connecting the b-wave and the d-wave has a negative slope. Therefore, it is generally well known that the line connecting the b-wave and the d-wave rises if the blood vessels are soft such as in adults in their twenties. On the other hand, line connecting the b-wave and the d-wave has a negative slope if the blood vessels are hard, such as in elderly persons in their seventies. That is, the more the line connecting the b-wave and the d-wave decreases, the greater the possibility of arterial sclerosis.

\subsubsection{Relationship between resultant bloodstream sounds and SDPTG}

The SDPTG indicates the acceleration of the bloodstream in a local domain. In contrast, in this experiment, our microphone sensors recorded low-frequency air vibration during the dilation and contraction of blood vessels, which are changes induced by the acceleration of the bloodstream in a local domain, as in Fig. 5. That is, the resultant bloodstream sounds (Fig.7(k)) and the SDPTG obtained using a pulse wave sensor machine for the same human subject (Fig.18(b)) have an analogous relationship in Fig.18 because the principle is almost the same. Moreover, it is determined that the line connecting the b-wave and the d-wave rises from Figs.18(a) and 19. On the other hand, it is determined that the line connecting the b-wave and the d-wave decreases from Figs.20(a) and (b).

Also, it is natural that the a-wave of two human subjects in their seventies (Figs.20(a) and (b)) lower than human subject in their twenties (Figs.18(a) and 19) because the a-wave expresses the degree of aging of blood vessels. As a result, in the case of human subjects in their twenties, the value of $b / a$ is large and $d / a$ is small. On the other hand, in human subjects in their twenties, the opposite is true. These results are the same as the general view.

Thus, we believe that we can also detect the hardness of blood vessels and predict arterial sclerosis from the bloodstream sounds that are separated by the proposed method. Through these facts, the results in Fig.7(k) are proven to be bloodstream sounds.

\section{Conclusion}

We proposed a robust method of separating heart, breathing, and bloodstream sounds from biosignals using microphone sensors. We solved the ICA problem related to considerable reverberation and the problem of initial-value dependence and noise in the EM algorithm by using 2-ch ICA to reduce noise and using the Dirichlet distribution. As a result, we obtained each sound robustly. In addition, we used a bandpass filter to obtain heart and breathing sounds, and in this way were able to obtain them from any biosignals. That is, we demonstrated that the proposed method is more robust than the existing method [7], [22]. We consider that IHB and SAS can be detected by analyzing S1 and S2 and inhalation and exhalation, using an autocorrelation algorithm. That is, the patients might have these diseases if the periodicity of the autocorrelation is varied. This must be confirmed in our future work.

In this study, IHB, arterial sclerosis and SAS were the targets, so the bandpass filter was robust at finding $\mathrm{S} 1$ and $\mathrm{S} 2$, and inhalation and exhalation. However, the use of the bandpass filter may not be suitable for other illnesses such as heart murmurs and accessory lung sounds, because this algorithm may remove information on heart murmur and accessory lung sounds depending on how the range of frequency is selected. Hence, we must increase the accuracy of separation. Therefore, we plan to add more microphone sensors to obtain more biosignal information and to use the wavelet algorithm. Separating heart, breathing, and bloodstream sounds without the bandpass filter may be possible, but noise is increased by the addition of microphone sensors. Therefore, we must formulate an algorithm to reduce the noise. We also plan to exploit the sparseness of biosignals. In particular, heart and lung sounds are constructed from signals that have a distinctive time-frequency structure. Thus, the separation of heart and breathing sounds and noise from biosignals may be possible by using basio that can briefly combine each component. These tasks are also our future work.

\section{Acknowledgment}

This research was supported by Semiconductor Technology Academic Research Center (STARC).

\section{References}

[1] M. T. Pourazad, Z. Moussavi and G. Thomas: Heart sound cancellation from lung sound recordings using time-freuency filtering, Med. Biol. Eng. Comput, Vol. 44, No. 3, pp. 216-225, 2006.

[2] K. Usman, M. A. Sadiq, H. Juzoji and I. Nakajima: A study of heartbeat sound separation using independent component analysis technique, Proc. HEALTHCOM, pp. 92-95, Jun. 2004.

[3] J.-C. Chien, M.-C. Huang, Y.-D. Lin and F.-C. Chong: A study of heart sound and lung sound separation by independent component analysis technique, Proc. EMBS, pp. 5708-5711, Aug. 2006.

[4] M. T. Pourazad, Z. Moussavi, F. Farahmand and R. K. Ward: Heart sounds separation from lung sounds using independent component analysis, Proc. EMBS, pp. 2736-2739, Jan. 2006.

[5] K. Hatta, M. Tada, H, Nakamura and M. Yoshida: The development of the separation system of the cardiac sound and respiratory sound by Independent Component Analysis, IEICE technical report. ME and bio cybernetics, Vol. 103, No. 731, pp. 39-44, 2004. 
[6] T. Tsalaile, S. M. Naqvi, K. Nazarpour, S. Sanei and J. A. Chambers: Blind source extraction of heart sound signals from lung sound recordings exploiting periodicity of the heart sound, Proc. ICASSP, pp. 461-464, Mar. 2008.

[7] A. K. Khan, T. Onoue, K. Hashiodani, Y. Fukumizu and H. Yamauchi: Signal and noise separation in medical diagnostic system based on independent component analysis, Proc. APCCAS, pp. 812-815, Dec 2010.

[8] T.-W. Lee (Ed.): Independent Component Analysis Theory and Applications, Kluwer, 1998.

[9] T. Hiekata, T. Morita, Y. Ikeda, H. Hashimoto, R. Zhang Y. Takahashi, H. Saruwatari and K. Shikano: Multiple ICA-based real-time blind source extraction applied to handy size microphone, Proc. ICASSP, pp. 121-124, Apr. 2009.

[10] S. Haykin (Ed.): Unsupervised Adaptive Filtering, Vol. 1, Blind Source Separation, Wiley, 2000.

[11] A. Hyvärinen, J. Karhunen and E. Oja (Eds.): Independent Component Analysis, Wiley, 2001.

[12] A. Cichocki and S. Amari (Eds.): Adaptive Blind Signal and Image Processing, Wiley, 2002.

[13] S. Makino, T.-W. Lee and H. Sawada (Eds.): Blind Speech Separation, Springer, 2007.

[14] H. Sawada, S. Araki and S. Makino: Underdetermined convolutive blind source separation via frequency bin-wise clustering and permutation alignment, IEEE. Trans. Audio, Speech and Lang. Process, Vol. 19, No. 3, pp. 516-527, 2011.

[15] S. Araki, H. Sawada, R. Mukai and S. Makino: Underdetermined blind sparse source separation for arbitrarily arranged multiple sensors, Signal Process, Vol. 87, No. 8, pp. 1833-1847, 2007.

[16] N. Roman, D. Wang and G. Brown: Speech segregation based on sound localization, J. Acoust. Soc. Am, Vol. 114, No. 4, pp. 2236-2252, 2003.

[17] M. Aoki, M. Okamoto, S. Aoki, H. Matsui, T. Sakurai and Y. Kaneda: Sound source segregation based on estimating incident angle of each frequency component of input signals acquired by multiple microphones, Acoust. Sci. Technol, Vol. 22, No. 2, pp. 149-157, 2001.

[18] H. Sawada, S. Araki and S. Makino: A two-stage frequency-domain blind source separation method for underdetermined convolutive mixtures, Proc. WASPAA, pp. 139-142, Oct. 2007.

[19] M. I. Mandel, D. P. W. Ellis and T. Jebara: An EM algorithm for localizing multiple sound sources in reverberant environments, in Advances in Neural Information Processing Systems 19, B. Schölkopf, J. Platt and T. Hoffman (Eds.), pp. 953-960, 2007.

[20] Y. Izumi, N. Ono and S. Sagayama: Sparseness-based 2ch BSS using the EM algorithm in reverberant environment, Proc. WASPAA, pp. 147-150, Oct. 2007.

[21] M. I. Mandel, R. J. Weiss and D. P. W. Ellis: Model-based expectation maximization source separation and localization, IEEE Trans. Audio,
Speech and Lang. Process, Vol. 18, No. 2, pp. 382-394, 2010.

[22] K. Hashiodani, T. Onoue, S. Takada, Y. Fukumizu, H. Yamauchi, Y. Kurumi and T. Tani: Biosignals separation method for medical diagnostic system, Proc. ISMICT, pp. 156-159, Mar. 2011.

[23] K. Matsuoka and S. Nakashima: Minimal distortion principle for blind source separation, Proc. ICA, pp. 722-727, Dec. 2001.

[24] H. Sawada, S. Araki and S. Makino: Measuring dependence of bin-wise separated signals for permutation alignment in frequency-domain BSS, Proc. ISCAS, pp. 3247-3250, May. 2007.

[25] C. M. Bishop (Ed.): Pattern Recognition and Machine Learning, Springer, 2008.

[26] S. Ikeda and N. Murata: A method of ICA in time-frequency domain, Proc. ICA, pp. 365-371, Jan. 1999.

[27] [Online] http://www.geocities.jp/ncn31_31/resp.htm, the homepage of study support of breathing sound.

[28] [Online] http://nishidaiin.com/ptg.htm, the homepage of second derivations of photoplethysmogram.

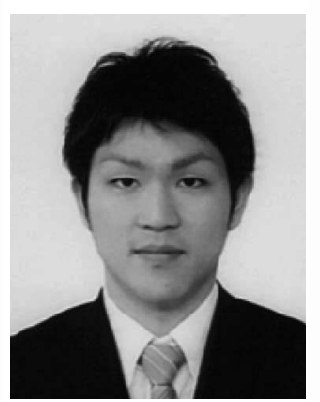

Kenji Hashiodani received his B.E. degree from the Department of VLSI System Design, Ritsumeikan University, Shiga, Japan, in 2010. He is currently a ME. student in the Graduate School of Science and Engineering, Ritsumeikan University. His research interests include signal processing, medical engineering, and pattern recognition. $\mathrm{He}$ is a member of IEEE and Acoustical Society of Japan.

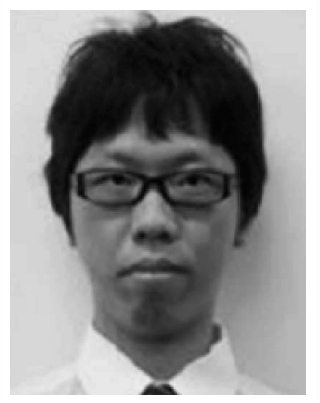

Shinich Takada received his B.E. degree from the Department of VLSI System Design, Ritsumeikan University, Shiga, Japan, in 2011. He is currently a M.E. student in the Graduate School of Science and Engineering, Ritsumeikan University. His research interests include sound signal processing, medical engineering, and pattern recognition. $\mathrm{He}$ is a member of IEEE. 


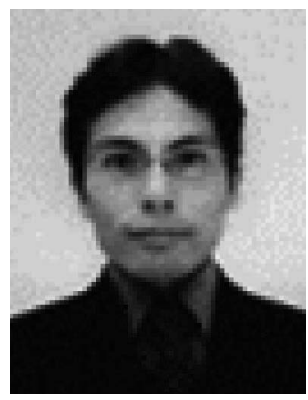

Yohei Fukumizu received his B.E. and M.E degrees in computer and systems engineering from Kobe University, Kobe, Japan, in 2001 and 2003, respectively, and his Ph.D. degree in computer engineering from Kobe University, Kobe, Japan, in 2007. He joined the Solutions Research Organization (SRO), the Integrated Research Institute (IRI), Tokyo Institute of Technology, Tokyo, Japan, as a postdoctoral researcher in 2007, where he participated in medical and biotechnology projects. In April 2008, he became an assistant professor in the Department of VLSI System Design, College of Science and Engineering, Ritsumeikan University, Shiga, Japan. His research interests focus on design methodologies of signal processing systems. He is a member of IEEE, IIEEJ, and IEICE.

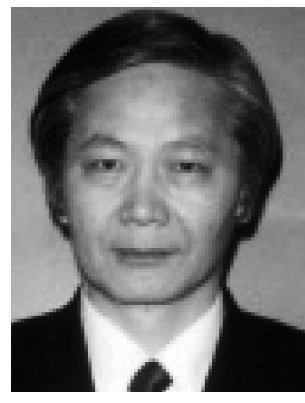

Hironori Yamauchi received his M.E and Dr.Eng. degrees from the University of Tokyo in 1975 and 1994, respectively. In 1975, he joined the Electrical Communications Laboratories of Nippon Telephone and Telegraph Public Corporation. He has been a Professor in the Faculty of Science and Engineering, Ritsumeikan University, since 1996. His research interests include pattern recognition, image signal processing, low power embedded systems architecture and related VLSI design. He is a fellow member of IEICE, and a member of IEEE, IPSJ, RISP

and IIEEJ.

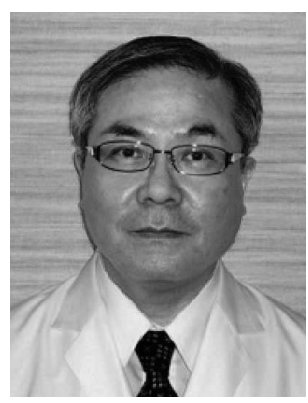

Yoshimasa Kurumi received his M.D. and $\mathrm{Ph} . \mathrm{D}$. degrees in medicine from Shiga University of Medical Science, Shiga, Japan, in 1981 , and 2000 , respectively. He became a resident at University Hospital, Shiga University of Medical Science, Shiga, Japan, in 1982. He became a surgeon at Murakami Memorial Hospital, Asahi University, Gifu, Japan, in 1983, and engaged in research on surgery. In 1985, he joined the Department of Surgery, Shiga University of Medical Science, where he is now an Associate Professor in the Department of Surgery and Clinical Professor at the General Surgery of University Hospital. His current research interests lie in the area of gastro-intestinal surgery and robotic surgery system. He is a Member of the Japanese Society of Surgery (JSS), Japanese Society of Gastro-Intestinal Surgery (JSGS) and Japanese Society of Endoscopic Surgery (JSES).

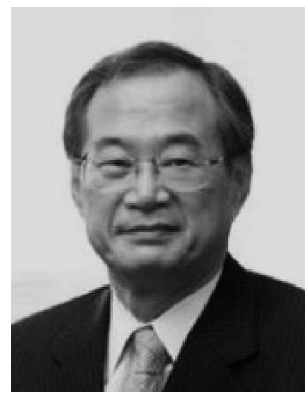

Tohru Tani received his M.D. degree in medicine from Kanazawa University, Ishikawa, Japan, in 1976, and Ph.D. degree in medicine from Shiga University of Medical Science, Shiga, Japan, in 1985. He became a resident at Toranomon Hospital, Tokyo, Japan, in 1976. He became a surgeon at Shiga University of Medical Science, Shiga, Japan, in 1985, and engaged in research on surgery. In 1998, he became an Associate Professor in the Department of Surgery, Shiga University of Medical Science, where he has been the Chief Professor in the Department of Surgery since 2001. His current research interests lie in the area of gastrointestinal surgery and robotic surgery. He is a Member of the Japanese Society of Surgery (JSS), Japanese Society of Gastro-Intestinal Surgery (JSGS) and Japanese Society of Artificial Organs

(Received October 3, 2011; revised January 9, 2012) 